\title{
Novel Experimental Preparation to Assess Electrocardiographic Imaging Reconstruction Techniques
}

\author{
Jake A Bergquist ${ }^{1,2,3}$, Brian Zenger ${ }^{1,2,3,4}$, Wilson W Good ${ }^{1,2,3}$, Lindsay C Rupp ${ }^{1,2,3}$, Laura R Bear ${ }^{5}$, \\ Rob S MacLeod ${ }^{1,2,3,4}$ \\ ${ }^{1}$ Scientific Computing and Imaging Institute, University of Utah, SLC, UT, USA \\ ${ }^{2}$ Nora Eccles Cardiovascular Research and Training Institute, University of Utah, SLC, UT, USA \\ ${ }^{3}$ Department of Biomedical Engineering, University of Utah, SLC, UT, USA \\ ${ }^{4}$ School of Medicine, University of Utah, SLC, UT, USA \\ ${ }^{5}$ IHU LIRYC, Université de Bordeaux, CRCTB Inserm U1045, Bordeaux, France
}

\begin{abstract}
Electrocardiographic imaging (ECGI) systems are still plagued by a myriad of controllable and uncontrollable sources of error, which makes studying and improving these systems difficult. To mitigate these errors, we developed a novel experimental preparation using a rigid pericardiac cage suspended in a torso-shaped electrolytic tank. The 256-electrode cage was designed to record signals $0.5-1.0 \mathrm{~cm}$ above the entire epicardial surface of an isolated heart. The cage and heart were fixed in a 192-electrode torso tank filled with electrolyte with predetermined conductivity. The resulting signals served as ground truth for ECGI performed using the boundary element method (BEM) and method of fundamental solutions (MFS) with three regularization techniques: Tikhonov zero-order (Tik0), Tikhonov second-order (Tik2), truncated singular value decomposition (TSVD). Each ECGI regularization technique reconstructed cage potentials from recorded torso potentials well with spatial correlation above 0.7 , temporal correlation above 0.8 , and root mean squared error values below $0.7 \mathrm{mV}$. The earliest site of activation was best identified by MFS using Tik0, which localized it to within a range of 1.9 and $4.8 \mathrm{~cm}$. Our novel experimental preparation has shown unprecedented agreement with simulations and represents a new standard for ECGI validation studies.
\end{abstract}

\section{Introduction}

ECGI is an established technique that uses subjectspecific geometry and body-surface electrocardiograms to reconstruct cardiac source signals. ECGI has high clinical utility, with several commercial systems on the market or in development. However, experimental and clinical validation studies for ECGI techniques have con- sistently shown significant errors from controllable and uncontrollable sources, including geometric registration, signal noise, conductivity uncertainty, choice of source model, and the inverse reconstruction method. [1,2] These diverse sources of error make it difficult to understand and interrogate ECGI approaches thoroughly, therefore there is a need for robust and controlled validation platforms with minimal sources of error.

To address this need, we developed a novel experimental preparation that limits sources of uncontrolled error. The preparation is similar to others used previously in that is uses a modified Langendorff perfused heart suspended inside a human shaped torso tank [3-5]. The torso tank allows control over several sources of error by utilizing a homogeneous isotropic volume conductor, recording high quality tank-surface ECG's with $\mathrm{Ag} / \mathrm{AgCl}$ electrodes in direct contact with the conductive media, and attaining expansive coverage with 192-electrodes spread across the tank surface. To further augment this preparation, we recently created a novel 256-electrode, pericardiac rigidcage electrode array that captures electrograms immediately adjacent to the heart surface. This cage array can be attached rigidly to the tank and samples evenly around the entire cardiac source, thus reducing both geometric and source sampling errors. Using this model, we have shown significant improvements in the upper error bounds of the forward problem, and leveraged the sampling of the cardiac cage to investigate atrial signal reconstruction. $[6,7]$ An outstanding opportunity was to demonstrate the utility of the preparation for the inverse problem and ECGI.

In this report, we introduce our novel experimental preparation and demonstrate its value for validating the accuracy of ECGI-based reconstruction of ventricular-paced beats with a range of inverse techniques. Specifically, we examined an ECGI formulation solved with the boundary element method (BEM) of the electrocardiographic 
forward problem and the method of fundamental solutions (MFS) with Tikhonov zero-order (Tik0), Tikhonov second-order (Tik2), and truncated singular value decomposition (TSVD) regularization techniques. We reconstructed the cardiac potentials on the rigid pericardiac cage with ECGI and assessed each formulation using standard global metrics, signal-based comparisons, and clinically relevant standards.

\section{Methods}

Experimental Preparation: The experimental preparation was based on a modified Langendorff system using a support animal to sustain the normal function of an isolated, perfused heart.[3,5] In brief, a heart was isolated from a donor animal and connected via arterial and venous access to a support animal. The isolated heart was perfused through the coronary vasculature via the aorta and blood was returned to the support animal via a suction pump in the right ventricle. The isolated heart was attached to a rigid gantry, which could be precisely lowered and fixed in the torso tank. Registration points were recorded using a Microscribe digitizer throughout the experiment to mark the location of the recording electrodes. All experiments were performed under deep anesthesia and approved by the Institutional Animal Care and Use Committee of the University of Utah according to the Guide for the Care and Use of Laboratory Animals.

Recording Arrays: We used two electrical recording arrays in this study, as shown in Figure 1. The first was the novel cage array, constructed from 256 silver-silver chloride electrodes evenly spaced around the centroid of the cage. The open structure of the cage allowed electrical current to flow unimpeded through the torso tank and generate ECG's at the electrodes embedded in the tank surface. The cage completely enclosed the beating heart with electrodes $0.5-1.0 \mathrm{~cm}$ from the epicardial surface.

The rigid cage and the Langendorff perfused heart were then suspended in the torso tank, which has been used in previous studies and was modeled after an adolescent human torso. The tank contains 192 embedded silver-silver chloride electrodes evenly spaced around the torso surface. The tank was filled with electrolytic fluid $(500 \Omega \cdot \mathrm{cm})$, which mimics the average conductivity of the human torso. Signals were acquired using a custom A/D multiplexer at $1 \mathrm{kHz}$ with a bandpass filter at 0.3 to $500 \mathrm{~Hz}$ and a notch filter at $60 \mathrm{~Hz}$. Signals were postprocessed and annotated using methods described previously. [8]

Beat Morphologies: For this study, we selected beats during subepicardial pacing from anterior and posterior left ventricular free wall using transmural plunge needle electrodes. For each pacing site, 40 individual beats were identified, processed, and used to compare reconstruction techniques.

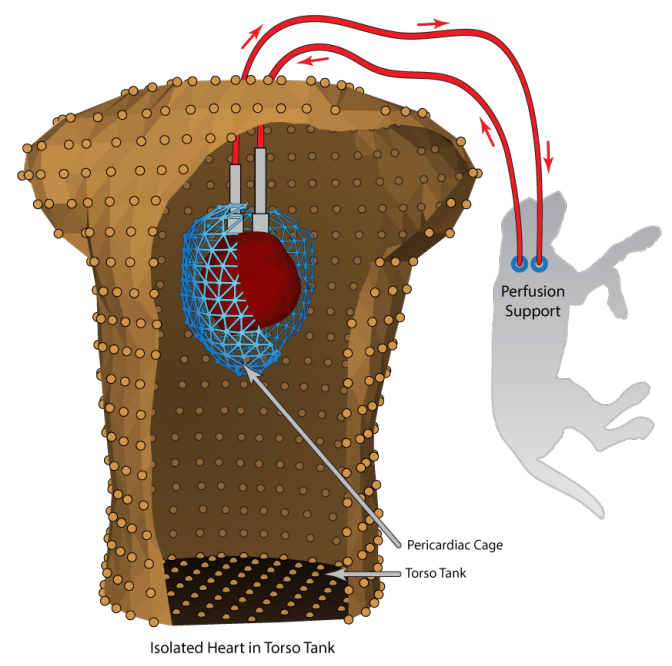

Figure 1. Schematic of experimental preparation with isolated heart, surrounded by the pericardiac cage and submerged in the electrolytic torso tank. See text for details.

Inverse Methods: We applied four common inverse formulations to calculate cage potentials: MFS with Tik0 regularization and BEM with Tik0, Tik2, and TSVD regularization. These inverse methods were implemented in the SCIRun problem-solving environment using the Forward/Inverse Toolkit and MATLAB.[9] For the BEM regularizations, the L-curve corner was used to select the regularization parameter $\lambda$ (Tik0 and Tik2) or the level of truncation (TSVD). For the MFS regularization with Tik0, we used the CRESO method to determine $\lambda$. The result was four inverse pipelines: BEM with Tik0, BEM with Tik2, BEM with TSVD, and MFS with Tik0.

Evaluation Metrics: To assess the accuracy of the inverse solutions, we calculated three standard statistical measures: RMSE, spatial correlation (SC), and temporal correlation (TC) according to the equations below. Here $\Phi(k, n)$ is a $K \times N$ matrix of measured $\left(\Phi_{m}\right)$ or calculated $\left(\Phi_{c}\right)$ potentials at $K$ electrodes on the cage for $N$ time instances. To calculate spatial or temporal correlation, we constructed zero mean vectors of either time signals on each electrode $\left(\phi^{t}=\Phi(k,:)-\overline{\Phi(k,:)}\right)$ or surface potential distributions at each time instance $\left(\phi^{s}=\Phi(;, t)-\overline{\Phi(:, t)}\right)$ respectively.

$$
\begin{aligned}
\mathrm{RMSE}= & \sqrt{\frac{\sum_{k=1}^{K} \sum_{n=1}^{N}\left(\left(\Phi_{c}(k, n)-\Phi_{m}(k, n)\right)^{2}\right.}{N \cdot K}} \\
\mathrm{SC} & =\frac{1}{N} \sum_{n=1}^{N} \frac{\phi_{m}^{s}(n)^{T} \phi_{c}^{s}(n)}{\left\|\phi_{m}^{s}(n)\right\| \cdot\left\|\phi_{c}^{s}(n)\right\|} \\
\mathrm{TC} & =\frac{1}{K} \sum_{k=1}^{K} \frac{\phi_{m}^{t}(k)^{T} \phi_{c}^{t}(k)}{\left\|\phi_{m}^{t}(k)\right\| \cdot\left\|\phi_{c}^{t}(k)\right\|}
\end{aligned}
$$


Previous studies have demonstrated that global statistics can be misleading.[1] To address this shortcoming, we implemented additional metrics: 1) the average magnitude of the spatial potential gradient at the peak of the RMS signal; 2) the activation localization error between the earliest computed and measured activation sites, and 3) a spatial correlation of reconstructed and measured activation maps.

\section{Results}

Table 1 contains global statistics for all inverse approaches; the overall performances of most were comparable. Mean RMSE values, spatial correlation, and temporal correlation varied from $0.52-0.65 \mathrm{mV}, 0.77-0.88$, and $0.80-0.95$, respectively. Across most global metrics, MFS with Tik0 performed consistently better with higher spatial and temporal correlations and slightly lower RMSE values. Correlations for activation maps ranged from 0.50 0.84 , with MFS Tik0 performing best. Figure 2 contains samples of the best and worst inverse reconstructions with respect to spatial correlation.

The additional metrics also revealed differences in reconstruction performance. For example, the mean magnitudes of the measured gradient were $0.095 \pm 0.10 \mathrm{mV} / \mathrm{mm}$ and $0.073 \pm 0.065 \mathrm{mV} / \mathrm{mm}$ for the first and second ventricularly paced beat morphologies, respectively. BEM Tik0 and TSVD preserved these gradients better than BEM Tik2 or MFS Tik0. The localization error was slightly lower with BEM Tik0 and TSVD, both identifying the earliest site of activation within $2.0-3.6 \mathrm{~cm}$.

\section{Discussion}

In this study, we reported a new experimental preparation with a rigid pericardiac cage recording array and showed its utility as a validation platform for ECGI. The preparation successfully attenuated many familiar sources of error, including geometry, signal noise, and conductivity. The recorded signals had both high spatial resolution and extensive coverage. We selected ventricular-paced beats for their simplicity, wide use for validating ECGI, and their clinical relevance. We found that most of the inverse methods performed similarly based on global metrics, corroborating previous studies using simulated and experimental datasets. However, to our knowledge, the ECGI performance presented here was the highest published with an experimental validation model. These results must be carefully interpreted because the signals were recorded just above the heart surface and not directly on the epicardium. The cage possessed substantial advantages, such as uniform coverage over the entire heart, and its robustness to cardiac motion artifacts.

We assessed the reconstruction results with specialized, clinically driven metrics, for example an assessment of the average spatial gradient at the peak of the QRS. Accurate gradient reconstruction is essential to correctly identify the direction of wave propagation and activation development. BEM Tik0 performed well at reconstructing such gradients, which is expected because the Tik0 regularization smooths the signal based on amplitudes. In contrast, Tik2 smooths based on the spatial derivative and limits the gradients between neighboring sites. BEM Tik0 performed the best at localizing the earliest site of activation, even if this error was larger than that reported in other ECGI systems. We anticipated this result because of the spacing between the pericardial cage and the epicardial surface. We could identify best-case scenarios in which the experimental vs. computed locations were a perfect match. Despite the superior spatial and temporal correlation values of the MFS Tik0 technique, it did not perform well on the other signal or clinically driven metrics.

In summary, our experimental preparation has several unique and compelling design features that control various components of error related to the inverse problem and ECGI. We can examine the inverse problem in unprecedented detail in an environment in which many of the common errors plaguing ECGI are controlled. The rigid pericardiac cage geometry limits the amount of geometric error, and the homogeneous torso tank and embedded electrode arrays give us adequate sampling and high-quality signal recordings while simplifying the volume conductor models. We recognize that this preparation fails to completely represent human anatomy or physiology; however, the resulting datasets can be immensely valuable in testing fundamental characteristics related to ECGI and practical applications. Future studies will further refine the experimental model to perfect the validation platform and use it in a variety of ECGI and clinically driven contexts.

\section{Acknowledgments}

Support for this research came from the NIHNIGMS Center for Integrative Biomedical Computing (www.sci.utah.edu/cibc), NIH NIGMS grants P41 GM103545 and R24 GM136986 and the Nora Eccles Treadwell Foundation for Cardiovascular Research.

\section{References}

[1] Bear LR, Cheng LK, LeGrice IJ, Sands GB, Lever NA, Paterson DJ, Smaill BH. Forward problem of electrocardiography: is it solved? Circulation Arrhythmia and Electrophysiology 2015;8(3):677-684.

[2] Cluitmans M, Brooks DH, MacLeod R, Dössel O, Guillem MS, van Dam PM, Svehlikova J, He B, Sapp J, Wang L, et al. Validation and opportunities of electrocardiographic imaging: from technical achievements to clinical applications. Frontiers in Physiology 2018;9:1305. 
Table 1. Reconstruction accuracy statistics: RMSE (mV), spatial correlation (SC), temporal correlation (TC), localization error (LE) (cm), activation map spatial correlation $\left(\mathrm{SC}_{\mathrm{act}}\right)$, mean gradient magnitude at RMS peak $(\mathrm{Grad})(\mathrm{mV} / \mathrm{mm}) \mathrm{c} . \mathrm{B}_{\mathrm{tik} 0}$ : BEM Tik0, $\mathrm{B}_{\mathrm{tik} 2}: \mathrm{BEM} \mathrm{Tik2,}_{\mathrm{tsvd}}: \mathrm{BEM} \mathrm{TSVD,}_{\mathrm{tik0}}$ : MFS Tik0. Statistics are shown as mean over all 40 beats per morphology \pm one standard deviation. Two pacing locations, one on the anterior (VP1) and one on the posterior (VP2) left ventricular free wall.

\begin{tabular}{|c|c|c|c|c|c|c|c|}
\hline \multirow[b]{3}{*}{ D } & & RMSE (mV) & $\mathrm{SC}$ & $\mathrm{TC}$ & $\mathrm{LE}(\mathrm{cm})$ & $\mathrm{SC}_{\mathrm{act}}$ & Grad (mV/mm) \\
\hline & VP 1 & $0.55 \pm 0.043$ & $0.79 \pm 0.028$ & $0.88 \pm 0.011$ & $3.6 \pm 1.4$ & $0.60 \pm 0.18$ & $0.082 \pm 0.049$ \\
\hline & VP 2 & $0.67 \pm 0.011$ & $0.77 \pm 0.013$ & $0.82 \pm 0.0043$ & $2.0 \pm 0.55$ & $0.55 \pm 0.10$ & $0.082 \pm 0.061$ \\
\hline \multirow{2}{*}{ 胥 } & VP 1 & $0.52 \pm 0.041$ & $0.88 \pm 0.031$ & $0.95 \pm 0.012$ & $4.7 \pm 2.4$ & $0.81 \pm 0.18$ & $0.053 \pm 0.028$ \\
\hline & VP 2 & $0.62 \pm 0.011$ & $0.85 \pm 0.0089$ & $0.86 \pm 0.00039$ & $2.6 \pm 1.5$ & $0.72 \pm 0.10$ & $0.054 \pm 0.033$ \\
\hline \multirow{2}{*}{ D } & VP 1 & $0.56 \pm 0.043$ & $0.77 \pm 0.029$ & $0.87 \pm 0.0096$ & $3.6 \pm 1.5$ & $0.59 \pm 0.15$ & $0.088 \pm 0.048$ \\
\hline & VP 2 & $0.67 \pm 0.011$ & $0.78 \pm 0.013$ & $0.80 \pm 0.0050$ & $2.0 \pm 0.55$ & $0.50 \pm 0.12$ & $0.085 \pm 0.055$ \\
\hline \multirow{2}{*}{$\sum^{\frac{0}{3}}$} & VP 1 & $0.49 \pm 0.041$ & $0.90 \pm 0.012$ & $0.95 \pm 0.0097$ & $5.2 \pm 2.3$ & $0.84 \pm 0.18$ & $0.055 \pm 0.029$ \\
\hline & VP 2 & $0.60 \pm 0.010$ & $0.87 \pm 0.0083$ & $0.86 \pm 0.0036$ & $3.3 \pm 0.36$ & $0.77 \pm 0.058$ & $0.050 \pm 0.035$ \\
\hline
\end{tabular}
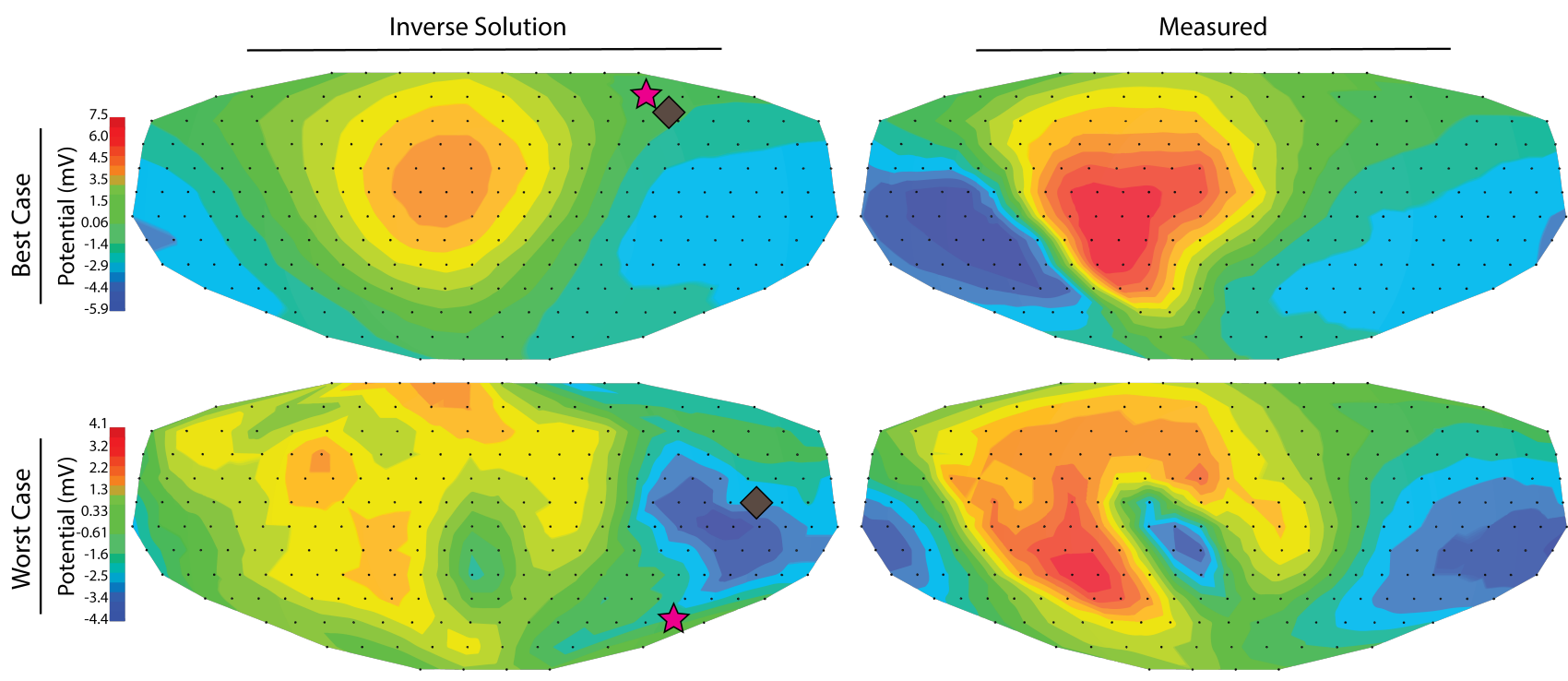

Figure 2. Identified best and worst case potential reconstructions on the pericardial surface at the peak of the QRS. Best case construction $(\mathrm{SC}=$ 0.91 ) used MFS Tik0 and the worst case $(\mathrm{SC}=0.65)$ used BEM TSVD. The pink star corresponds to the measured earliest site of activation. The brown diamond corresponds to the computed earliest site of activation

[3] Shome S, Macleod R. Simultaneous high-resolution electrical imaging of endocardial, epicardial and torso-tank surfaces under varying cardiac metabolic load and coronary flow. In International Conference on Functional Imaging and Modeling of the Heart. Springer, 2007; 320-329.

[4] Coll-Font J, Brooks DH. Tracking the position of the heart from body surface potential maps and electrograms. Frontiers in Physiology 2018;9:1727.

[5] Punske BB, Lux RL, MacLEOD RS, Fuller MS, Ershler PR, Dustman TJ, Vyhmeister Y, Taccardi B. Mechanisms of the spatial distribution of qt intervals on the epicardial and body surfaces. Journal of Cardiovascular Electrophysiology 1999; 10(12):1605-1618.

[6] Bergquist JA, Good WW, Zenger B, Tate JD, MacLeod RS. The Cardiac Forward Problem: A Benchmark Study. In Preparation 2020;.

[7] Bergquist J, Good W, Zenger B, Tate J, MacLeod R. Optimizing the reconstruction of cardiac potentials using a novel high resolution pericardiac cage. In Computing in Cardiol- ogy 2019, volume 46. 2019; 1-4.

[8] Rodenhauser A, Good WW, Zenger B, Tate J, Aras K, Burton B, MacLeod RS. Pfeifer: Preprocessing framework for electrograms intermittently fiducialized from experimental recordings. The Journal of Open Source Software 2018; 3:472.

[9] Burton B, Tate J, Erem B, Swenson D, Wang D, Brooks D, van Dam P, MacLeod R. A toolkit for forward/inverse problems in electrocardiography within the SCIRun problem solving environment. In Proceedings of the IEEE Engineering in Medicine and Biology Society 33rd Annual International Conference. IEEE Eng. in Med. and Biol. Soc., 2011; $1-4$.

Address for correspondence:

Jake Bergquist, jbergquist@ sci.utah.edu, University of Utah 72 Central Campus Dr, Salt Lake City, UT 84112 